\title{
Index-Based Dietary Patterns and the Risk of Prostate Cancer among Iranian Men
}

\author{
Matin Ghanavti ${ }^{1}$, Mehdi Movahed ${ }^{2}$, Bahram Rashidkhani ${ }^{2}$, Afshin Rakhsha ${ }^{3}$, \\ Ehsan Hejazi ${ }^{4 *}$
}

\begin{abstract}
Background and objective: The second most common cancer in men after lung cancer is prostate cancer (PC). Previous studies assessed the association between food items or food groups and the risk of PC, but diet quality indices are unique approaches to study any relations between diet and disease. Our objective was to investigate the effect of healthy eating index (HEI-2010) and Mediterranean-Style Dietary Pattern Score (MSDPS) on PC risk. Methods: In this case-control study, we recruited 97 patients with MS and 205 control subjects . Dietary intake was evaluted using a valid and reliable food frequency questionnaire. The HEI and MSDPS were calculated. Logistic regression was used to evaluate the relationship between HEI and MSDP scores and PC risk after adjusting the confounders. Results: In comparison to controls, cases had lower score on HEI (61 vs. 70.07; $\mathrm{P}<0.001$ ), and higher score on MSDP (26.20 vs. 24.49; $\mathrm{P}=0.44)$. After comparing the highest and the lowest tertile of HEI, we observed a significant decreasing trend in the risk of PC ( $\mathrm{p}$ for trend $<0.001$ ). Conclusion: Our findings suggested that a high quality diet, according to HEI, may decrease the risk of PC.
\end{abstract}

Keywords: Healthy Eating Index- Mediterranean-Style Dietary Pattern Score- diet quality- prostate cancer

Asian Pac J Cancer Prev, 20 (5), 1393-1401

\section{Introduction}

Prostate cancer ( $\mathrm{PCa}$ ) is the most diagnosed malignancy of men in Western countries (Grubb and Kibel, 2007). The incidence of PC in Asian countries, including Iran, is lower than that in Western populations (Hosseini et al., 2010). Various factors, including genetic factors, screening methods, lifestyle, and diet contribute to this difference (Baade et al., 2009; Zhang et al., 2011). Numerous studies focused on the effects of a nutrient on PCa. Some studies reported the effects of single food or nutrient on PCa (Giovannucci et al., 2002; Chavarro et al., 2007; Park et al., 2007). Dietary components are consumed in combination, affecting the absorption and bioavailability of each other (Michels and Schulze, 2005). Hence, identifying individual effect of dietary components is very difficult.

Dietary patterns allow to discover the link between the overall diet and the risk of disease (Moeller et al., 2007). Two of the most popular indices for measuring healthy eating patterns are The Healthy Eating Index (HEI) 2010 and The Mediterranean-Style Dietary Pattern Score (MSDPS). HEI 2010 examines the diet quality based on its conformance with Food Guide Pyramid and Dietary
Guidelines (Guenther et al., 2013; Guenther et al., 2014) and MSDPS examines adherence to the Mediterranean dietary pattern (Rumawas et al., 2009a; Rumawas et al., 2009b). High adherence to HEI and MSDPS has been associated with decreased risk of total mortality and cancers (Trichopoulou et al., 2000; Mitrou et al., 2007; Reedy et al., 2008). However, no study has investigated the association between PCa among Iranian men and HEI 2010 and MSDPS yet. So, the aim of this study was to determine the relation between performance on HEI 2010 and MSDPS and PC risk among Iranian male.

\section{Materials and Methods}

\section{Participants and methods \\ Subjects}

This hospital based case-control study, carried out in Tehran (the capital of Iran). Participants included 140 men with a confirmed histological diagnosis of PC in the preceding six months, who were referred to Shahid Labafi and Modares hospitals. Out of these 140 patients, 135 were selected based on our defined inclusion and exclusion criteria. The inclusion criteria were as follows: 1) aged between 40 and 80 years old; 2) did not undergo

${ }^{1}$ Student Research Committee, ${ }^{2}$ Community Nutrition Department, Faculty of Nutrition Sciences and Food Technology, National Nutrition and Food Technology Research Institute (WHO Collaborating Center), ${ }^{3}$ Shohada-e-Tajrish Hospital, Department of Radiation Oncology, Faculty of Medicine, ${ }^{4}$ Department of Clinical Nutrition and Dietetics, School of Nutrition Sciences and Food Technology, Shahid Beheshti University of Medical Sciences, Tehran, Iran. *For Correspondence: ehsanhejazi@gmail.com 
any changes in his diet since diagnosis; 4) be willing to participate in the study; 5) being a male. Based on our inclusion criteria, 100 men with PC were selected as case group (participation rate $=71 \%$ ). Based on inclusion criteria, 100 men with PC selected as case group. In addition, 205 patients who were admitted to the same hospitals (due to ear, nose, or eye disorder, appendicitis or general surgery) without any special diets were selected as the control group. Individuals in case and control groups were matched in terms of age (with a ten-year-interval). At the end, 3 patients in the case group whose their log scale of total energy intake were either $>3$ SD or $<3$ SD from the mean were excluded from the study. Therefore, 97 cases and 205 controls were underwent further analysis. All participants were interviewed to obtain needed information, including history of diabetes, hypertension, smoking status, alcohol use, and marital status. The weight of each participant with the least amount of clothing and a sensitivity of 100 gram was obtained by a digital scale, and the height of each of them was measured without shoes with a sensitivity of $0.1 \mathrm{~cm}$. We measured waist circumference through this procedure: find the top of hip and the bottom of ribs, place the tap measure midway between these points, and wrap it around waist. The study was approved by Ethics Committee of Shahid Beheshti University of Medical Sciences.

\section{Dietary assessment}

Data on usual dietary intake of participants over the past year were collected through a valid and reliable semi-quantitative food frequency questionnaire (148-item FFQ) (Esfahani et al., 2010). All participants were asked to record food consumption frequency of every food item with standard serving size per day, per week, per month, and per year. The amount of each food item was convert to gram per day using the household scales (Ghaffarpour et al., 1999). Analyses of energy and nutrients were carried out using the USDA FCT (McGuire, 2011). However, for some dairy products such as Kashk, vetch, wild plum, mint, sweet canned cherry, and sour cherry that are not listed in the USDA FCT, Iranian FCT was used alternatively (Movahedi and Roosta, 2000). In order to calculate healthy eating components, we used national nutrient database to convert values obtained from FFQ to cup or ounce equivalents per $1000 \mathrm{kcal}$ (Bowman et al., 2014).

\section{Calculation of the Healthy Eating Index-2010}

Healthy eating index-2010 (HEI-2010), as a measure of quality of life, was designed according to recommendations presented by the dietary guidelines for American 2010 (McGuire, 2011). The HEI is used to examine the relationships between diet and health-related outcomes. A summary of HEI-2010 components and their point values as well as its scoring standards are presented in Table 1. HEI-2010 includes 12 components divided into 9 adequacy components and 3 moderation components. Adequacy components consist of total fruit, whole fruit, total vegetables, greens and beans, whole grains, dairy, seafood and plant proteins, fatty acids, and total protein foods. Moderation components are refined grains, sodium, and empty calories (Guenther et al., 2013). All components scores were calculated based on their point values that is presented in Table 1. The participant with zero intake of adequacy components except fatty acids received a score of zero. For all adequacy components, the maximum number of points assigned to participants with intake at the level of the standard or higher. Other components scoring was carried out based on the standard for maximum score and minimum score of zero. Then, scores for the intake between the minimum and maximum standards were calculated proportionately.

\section{Calculation of the Mediterranean-Style Dietary Pattern} Score (MSDPS)

Mediterranean-Style Dietary Pattern Score (MSDPS) is consisted of 13 components based on Mediterranean pyramid. These 13 food groups are whole-grain cereals, fruits, vegetables, dairy products, wine, fish and other seafood, poultry, olives/legumes/nuts, potatoes and other starchy roots, eggs, sweets, meat, and olive oil (Rumawas et al., 2009a). In our study, only 12 components were available because participants did not respond to alcohol intake questions due to religious beliefs (Hosseini-Esfahani et al., 2010). With the exception of olive oil, each group was scored from 0 to 10 depending on the degree of correspondence with the recommendations. Olive oil was scored differently, score 10 was allocated to exclusive olive oil intake, score 5 to use of olive oil along with other vegetable oils, and score 0 to olive oil non-consumption. We also took into account the negative implications of overconsumption, defined as exceeding the recommended intake of foods in the Mediterranean diet pyramid. Overconsumption incurs a penalty by subtracting a point proportionally to the number of consumed servings that exceeded the recommended intake for that group. Finally for each participant, all 12 scores were summed up, and then were divided into proportion of total energy intake from Mediterranean diet in order to calculate the energy adjusted MSDPS that ranged from 0 (minimal adherence to Mediterranean pyramid recommendations) to 100 (maximal adherence) (Rumawas et al., 2009a).

\section{Statistical analysis}

Statistical analyses were conducted using SPSS version 19.0 (SPSS Inc., Chicago, IL, USA). Normal distribution of quantitative data was measured by the Kolmogorov-Smirnov test and normal distribution of qualitative data was measured by the Chi-square test. To compare differences between cases and controls, independent sample t-test and the Mann-whitney test were used for continuous variables and categorical variables, respectively.

Each of the density standards and the overall HEI score were categorized into tertiles based on the distribution among controls. We used binary logistic regression to estimate odds ratios and $95 \%$ confidence intervals for tertiles of each 12 component. Considered covariates included body mass index (continuous), waist circumstance, total energy intake (continuous), smoking status, and history of diabetes, and hypertension. Then, odds ratio was adjusted for this confounding factors. 


\section{Results}

Characteristics of 97 PC patients as cases and 205 controls are shown in Table 1. Cases and controls were not significantly different in terms of age, energy intake, alcohol use, and marital status, but they indicated significant differences in terms of BMI, waist circumference, history of diabetes, heart disease, hypertension, physical activity, and smoking status $(\mathrm{P}<0.05)$. Table 2 shows a comparison between the case (PC) and control groups based on the scores of HEI components and daily intake. Total HEI-2010 was significantly higher in the control group than the case group ( $<<0.001)$. The control group compared with the case group had higher intake and scores on components of whole fruit, total fruit, total vegetables, fatty acids $(\mathrm{p}<0.001)$, seafood, and plant protein $(\mathrm{p}=0.023)$. Sodium intake was significantly lower in the control group than that in the case group. Table 3 shows odds ratios (OR) and confidence intervals $(95 \% \mathrm{CI})$ for PC by HEI-2010 components as well as total HEI-2010 scores. After adjusting the impact of confounders (energy intake, BMI, waist circumstance, family history of diabetes and blood pressure and smoking status). Individuals in the highest tertile of total fruit intake $(\mathrm{OR}=0.14 ; 95 \% \mathrm{CI}=0.05-0.42)$, total vegetables $(\mathrm{OR}=0.23 ; 95 \% \mathrm{CI}=0.09-0.59)$, whole fruit $(\mathrm{OR}=0.15 ; 95 \% \mathrm{CI}=0.05-0.44)$, and fatty acids $(\mathrm{OR}=0.12 ; 95 \% \mathrm{CI}=0.03-0.37)$ showed a significant decrease in the risk of PC compared to those in the lowest tertile of intake. There was no difference in terms of MSDPS between case and control groups (Table 5).

Table 1. The Standard Scoring for HEI-2010

\begin{tabular}{|c|c|c|c|}
\hline HEI-2010 components & Maximum points & Standard for maximum score & Standard for minimum score of zero \\
\hline Total fruit ${ }^{1}$ & 5 & $\geq 0.8$ cup equiv. per $1,000 \mathrm{kcal}$ & No fruit \\
\hline Whole fruit ${ }^{2}$ & 5 & $\geq 0.4$ cup equiv. per $1,000 \mathrm{kcal}$ & No whole fruit \\
\hline Total vegetables ${ }^{3}$ & 5 & $\geq 1.1$ cup equiv. per $1,000 \mathrm{kcal}$ & No vegetables \\
\hline Greens and Beans ${ }^{3}$ & 5 & $\geq 0.2$ cup equiv. per $1,000 \mathrm{kcal}$ & No dark greens or beans and peas \\
\hline Whole Grains & 10 & $\geq 1.5 \mathrm{oz}$ equiv. per $1,000 \mathrm{kcal}$ & No whole grains \\
\hline Dairy $^{4}$ & 10 & $\geq 1.3$ cup equiv. per $1,000 \mathrm{kcal}$ & No dairy \\
\hline Total Protein Foods ${ }^{5}$ & 5 & $\geq 2.5 \mathrm{oz}$ equiv. per $1,000 \mathrm{kcal}$ & No protein foods \\
\hline Seafood and Plant Proteins ${ }^{6}$ & 5 & $\geq 0.8$ oz equiv. per $1,000 \mathrm{kcal}$ & No seafood and plant proteins \\
\hline Fatty Acids ${ }^{7}$ & 10 & $($ PUFAs + MUFAs) $/$ SFAs $\geq 2.5$ & PUFAs + MUFAs) $/$ SFAs $\leq 1.2$ \\
\hline Refined Grains ${ }^{8}$ & 10 & $\leq 1.8$ oz equiv. per $1,000 \mathrm{kcal}$ & $\geq 4.3$ oz equiv. per $1,000 \mathrm{kcal}$ \\
\hline Sodium & 10 & $\leq 1.1$ gram per $1,000 \mathrm{kcal}$ & $\geq 2.0$ grams per $1,000 \mathrm{kcal}$ \\
\hline Empty calories & 20 & $\leq 19 \%$ of energy & $\geq 50 \%$ of energy \\
\hline
\end{tabular}

1, includes fruit juice; 2, includes all forms except juice; 3, include any beans and peas; 4, includes all milk products, such, such as fluid milk, yogurt, cheese and fortified soy beverage; 5 , if total protein foods standard not met, beans and peas are included here; 6 , includes seafood, nuts, seeds, soy products (except beverages), beans, and peas; 7, ratio of PUFA+MUFA/ SFA; 8, calories from solid fats, alcohol, and added sugars

Table 2. Baseline Characteristics of PC Cases and Healthy Controls, in a Case Control Study of PC

\begin{tabular}{|c|c|c|c|}
\hline Characteristics & Case (97) & Control (205) & P-Value* \\
\hline Age (year) & $59.82(9.55)$ & $59.59(9.32)$ & 0.85 \\
\hline BMI $\left(\mathrm{kg} / \mathrm{m}^{2}\right)$ & $25.61(2.35)$ & $29.12(5.25)$ & $<0.001$ \\
\hline Waist circumstance $(\mathrm{cm})$ & $82.08(11.12)$ & $90.13(10.32)$ & $<0.001$ \\
\hline Energy (kcal/day) & $2837(925.78)$ & 2989 (938.74) & 0.18 \\
\hline Marital status & & & 0.1 \\
\hline Married & $90(92.8)$ & $167(81.5)$ & \\
\hline Not married & $7(7.2)$ & $38(18.5)$ & \\
\hline Smoking status & & & $<0.001$ \\
\hline Yes & $9(9.3)$ & $0(0.0)$ & \\
\hline No & $88(90.7)$ & $205(100)$ & \\
\hline Diabetes & & & $<0.001$ \\
\hline Yes & $8(8.2)$ & $0(0.0)$ & \\
\hline No & $89(91.8)$ & $205(100)$ & \\
\hline Hypertension & & & $<0.001$ \\
\hline Yes & $21(21.6)$ & $0(0.0)$ & \\
\hline No & $76(78.4)$ & $205(100)$ & \\
\hline
\end{tabular}

Data are presented as frequency (percentage) or mean(SD); P-value was estimated using $\chi 2$ or fisher test and t-test 
Table 3. A Comparison between the Case (PC Patients) and Control Groups Based on Daily Intake and Scores of the Healthy Eating Index (HEI) Components

\begin{tabular}{|c|c|c|c|c|c|c|c|}
\hline \multirow{2}{*}{$\begin{array}{l}\text { HEI-2010 } \\
\text { components }\end{array}$} & \multicolumn{4}{|c|}{ Intake per day } & \multicolumn{3}{|c|}{ HEI-2010 scores } \\
\hline & Unit & Case (97) & Control (205) & pvalue & Case (95) & Control (205) & pvalue \\
\hline HEI & & & & & $\begin{array}{c}619.37 \pm \\
(54.56-66.68)\end{array}$ & $\begin{array}{c}70.07 \pm \\
(64.35-76.70)\end{array}$ & $<0.001 * *$ \\
\hline Total fruit & Cup & $\begin{array}{c}1.190 .78 \pm \\
(0.67-1.71)\end{array}$ & $\begin{array}{l}1.68 \pm 0.88 \\
(1.11-2.14)\end{array}$ & $<0.001 *$ & $\begin{array}{l}4.18 \pm 1.59 \\
(4.21-5.00)\end{array}$ & $\begin{array}{l}4.84 \pm 0.62 \\
(5.00-5.00)\end{array}$ & $<0.001 *$ \\
\hline Whole fruit & Cup & $\begin{array}{l}1.16 \pm 0.76 \\
(0.64-1.66)\end{array}$ & $\begin{array}{l}1.64 \pm 0.86 \\
(1.08-2.09)\end{array}$ & $<0.001 *$ & $\begin{array}{l}4.43 \pm 1.51 \\
(5.00-5.00)\end{array}$ & $\begin{array}{c}4.94 \pm \\
(5.00-5.00)\end{array}$ & $<0.001 *$ \\
\hline Total vegetables & Cup & $\begin{array}{c}1.1 \pm 0.57 \\
(0.69-1.45)\end{array}$ & $\begin{array}{l}1.86 \pm 3.55 \\
(1.04-1.88)\end{array}$ & $<0.001 *$ & $\begin{array}{l}3.99 \pm 1.12 \\
(3.15-5.00)\end{array}$ & $\begin{array}{l}4.68 \pm 0.68 \\
(4.76-5.00)\end{array}$ & $<0.001 *$ \\
\hline Greens and Beans & Cup & $\begin{array}{l}0.24 \pm 0.22 \\
(0.10-0.31)\end{array}$ & $\begin{array}{l}0.47 \pm 3.40 \\
(0.08-0.30)\end{array}$ & 0.29 & $\begin{array}{l}3.75 \pm 1.45 \\
(2.66-5.00)\end{array}$ & $\begin{array}{c}3.5 \pm 1.62 \\
(2.01-5.00)\end{array}$ & 0.29 \\
\hline Whole Grains & $\mathrm{OZ}$ & $\begin{array}{l}1.36 \pm 1.56 \\
(0.31-1.91)\end{array}$ & $\begin{array}{c}1.1 \pm 1.31 \\
(0.41-1.20)\end{array}$ & 0.32 & $\begin{array}{c}5.81 \pm 3.75 \\
(2.07-10.00)\end{array}$ & $\begin{array}{l}5.39 \pm 3.18 \\
(2.76-8.06)\end{array}$ & 0.34 \\
\hline Dairy & Cup & $\begin{array}{l}0.83 \pm 0.40 \\
(0.58-1.03)\end{array}$ & $\begin{array}{l}0.83 \pm 0.41 \\
(0.54-1.11)\end{array}$ & 0.99 & $\begin{array}{l}6.12 \pm 2.42 \\
(4.49-7.92)\end{array}$ & $\begin{array}{l}6.15 \pm 2.61 \\
(4.17-8.54)\end{array}$ & 0.92 \\
\hline Total Protein Foods & $\mathrm{OZ}$ & $\begin{array}{l}1.63 \pm 0.63 \\
(1.12-2.01)\end{array}$ & $\begin{array}{l}1.72 \pm 0.70 \\
(1.23-2.10)\end{array}$ & 0.35 & $\begin{array}{l}3.18 \pm 1.10 \\
(2.25-4.03)\end{array}$ & $\begin{array}{l}3.31 \pm 1.13 \\
(2.46-4.20)\end{array}$ & 0.35 \\
\hline Seafood and Plant Proteins & $\mathrm{OZ}$ & $\begin{array}{l}0.52 \pm 0.42 \\
(0.22-0.71)\end{array}$ & $\begin{array}{l}0.46 \pm 0.56 \\
(0.12-0.63)\end{array}$ & $0.03 *$ & $\begin{array}{c}3.59 \pm 1.46 \\
(2.22-0)\end{array}$ & $\begin{array}{l}3.91 \pm 1.33 \\
(2.90-5.00)\end{array}$ & $0.023 *$ \\
\hline Fatty Acids & gr & $\begin{array}{c}1.21 \pm \\
(0.89-1.40)\end{array}$ & $\begin{array}{l}2.08 \pm 0.78 \\
(1.52-2.47)\end{array}$ & $<0.001^{*}$ & $\begin{array}{c}2.49 \pm 3.33 \\
(0-5.61)\end{array}$ & $\begin{array}{l}7.34 \pm 2.54 \\
(6.08-9.88)\end{array}$ & $<0.001^{*}$ \\
\hline Refined Grains & $\mathrm{OZ}$ & $\begin{array}{l}4.15 \pm 2.31 \\
(2.67-5.37)\end{array}$ & $\begin{array}{l}4.19 \pm 2.04 \\
(2.73-5.39)\end{array}$ & 0.53 & $\begin{array}{c}4.32 \pm 3.65 \\
(0-6.72)\end{array}$ & $\begin{array}{c}3.99 \pm 3.65 \\
(0-6.59)\end{array}$ & 0.44 \\
\hline Sodium & gr & $\begin{array}{c}107.6 \pm \\
(55.30-143.86)\end{array}$ & $\begin{array}{c}3.06 \pm \\
(1.62-3.31)\end{array}$ & $<0.001 *$ & $\begin{array}{c}0.27 \pm 1.57 \\
(0-0)\end{array}$ & $\begin{array}{c}2.95 \pm 3.72 \\
(0-6.59)\end{array}$ & $<0.001 *$ \\
\hline Empty calories & $\begin{array}{c}\% \text { of } \\
\text { energy }\end{array}$ & $\begin{array}{c}17.31 \pm 5.88 \\
(13.68-19.38)\end{array}$ & $\begin{array}{c}16.52 \pm 5.62 \\
(12.27-20.29)\end{array}$ & 0.29 & $\begin{array}{c}18.94 \pm 2.64 \\
(19.60-20)\end{array}$ & $\begin{array}{c}18.97 \pm 1.96 \\
(18.76-20.00)\end{array}$ & 0.74 \\
\hline
\end{tabular}

Value presented as mean \pm SD (IQR); * Significant difference (Mann-Whitney, p-value $<0.05) ; * *$ Significant difference (t-test, p-value $<0.05)$

\section{Discussion}

The associations between MSDPS and HEI 2010 and risk of PC were evaluated in this study. We found that participants with a higher adherence to HEI-2010 had a reduced risk of $\mathrm{PC}$. In contrast, no significant association was found between MSDPS and risk of PC. Only few studies examined the index-based patterns and risk of PC. Bosire et al., (2013) found that high AHEI-2010 and HEI- 2005 scores were associated with lower risk of total PC. The HEI-2010 seems to be more relevant than AHEI-2010 in decreasing cancer risk and mortality (Onvani et al., 2017). Differences between the two patterns may be due to this fact that HEI does not include alcoholic beverages and red and processed meat, whiles they are considered as AHEI components. According to previous studies, adherence to a Mediterranean dietary pattern was related to reduce risk of overall mortality and cancer incidence and mortality (Sofi et al., 2010; Verberne et al., 2010). However, several studies found no association between the Mediterranean diet score and PC (Tseng et al., 2004; Möller et al., 2013; Ax et al., 2014), our findings were consistent with those of other studies. The risk estimate is based on components of the Mediterranean diet pattern that is scored based on the intake of population distribution. The reported median intake in a Greek population is extremely higher than that in other populations listed here. The identified
Mediterranean dietary pattern in these populations were not extensively Mediterranean, which may explain the different findings. Findings on dietary pattern and $\mathrm{PC}$ are inconsistent. A number of studies found no association between specific dietary patterns and PC (Tseng et al., 2004; Wu et al., 2006; Muller et al., 2009); however; the other studies reported a positive association between a western dietary pattern and PC (Ambrosini et al., 2008). Individual component analyses were also performed, indicating significant inverse association between PC and seafood and plant protein as well as the ratio of total unsaturated/saturated fatty acids in HEI-2010 and the components of vegetables and fruits in both HEI-2010 and MED. Previous studies also found an inverse association between fish intake and PC risk (Augustsson et al., 2003; Ambrosini et al., 2008; Chavarro et al., 2008; Pham et al., 2009). Augustsson et al., (Augustsson et al., 2003) showed that eating fish 3 or more times per week could reduce risk of metastatic PC 44\% compared to its consumption less than 2 times per month. This association was also reported by Chavarro et al., (2008). A proposed mechanism for the protective effect of fish on $\mathrm{PC}$ is the long-chained $\omega$-3 fatty acids present in fish. Both EPA and DHA have been found to inhibit the biological activity of eicosanoids and androgens (Faust et al., 1989; Zaccheo et al., 1998), which could stimulate the growth of PC cells (Ghosh and Myers, 1997; Rose, 1997). The results of this study on the consumption of plant protein seem to 
Table 4. OR and 95\% Confidence Interval for Risk of PC Based on Tertiles of HEI-2010 Components and Total HEI-2010

\begin{tabular}{|c|c|c|c|c|c|c|}
\hline Variable in tertile & Case (n) & Control (n) & $\mathrm{OR}^{1}$ & CI & $\mathrm{OR}^{2}$ & $\mathrm{CI}$ \\
\hline \multicolumn{7}{|l|}{ HEI-2010 (SCORE) } \\
\hline Tertile $1(<66.08)$ & 70 & 68 & Ref & & Ref & \\
\hline Tertile2 (66.08-73.20) & 18 & 69 & 0.25 & $(0.13-0.47)$ & 0.19 & $(0.01-0.49)$ \\
\hline Tertile3 (>73.20) & 9 & 68 & 0.12 & $(0.059-0.27)$ & 0.063 & $(0.01-0.28)$ \\
\hline$P$ for trend & & & $<0.001$ & & $<0.001$ & \\
\hline \multicolumn{7}{|l|}{ Total fruit ${ }^{\mathrm{a}}$} \\
\hline Tertile $1(<1.22)$ & 59 & 66 & ref & & & \\
\hline Tertile2 (1.22-1.85) & 18 & 71 & 0.28 & $(0.15-0.53)$ & 0.11 & $(0.03-0.32)$ \\
\hline Tertile3 (>1.85) & 20 & 68 & 0.32 & $(0.17-0.60)$ & 0.14 & $(0.05-0.42)$ \\
\hline$P$ for trend & & & $<0.001$ & & $<0.001$ & \\
\hline \multicolumn{7}{|l|}{ Whole fruit ${ }^{\mathrm{a}}$} \\
\hline Tertile $1(<1.20)$ & 59 & 66 & Ref & & Ref & \\
\hline Tertile2 (1.20-1.80) & 19 & 70 & 0.3 & $(0.16-0.56)$ & 0.12 & $(0.04-0.36)$ \\
\hline Tertile3 (>1.80) & 19 & 69 & 0.3 & $(0.16-0.15)$ & 0.15 & $(0.05-0.44)$ \\
\hline $\mathrm{P}$ for trend & & & $<0.001$ & & $<0.001$ & \\
\hline \multicolumn{7}{|l|}{ Total vegetables ${ }^{\mathrm{a}}$} \\
\hline Tertile $1(<1.15)$ & 61 & 68 & Ref & & Ref & \\
\hline Tertile2 (1.15-1.73) & 25 & 68 & 0.41 & $(0.23-0.72)$ & 0.27 & $(0.11-0.67)$ \\
\hline Tertile3 (>1.73) & 11 & 69 & 0.17 & $(0.80-0.36)$ & 0.23 & $(0.09-0.59)$ \\
\hline $\mathrm{P}$ for trend & & & $<0.001$ & & 0.001 & \\
\hline \multicolumn{7}{|l|}{ Greens and Beans ${ }^{\mathrm{a}}$} \\
\hline Tertile $1(<0.10)$ & 23 & 68 & Ref & & Ref & \\
\hline Tertile2 (0.10-0.26) & 42 & 68 & 1.82 & $(0.99-3.35)$ & 2.55 & $(0.91-7.01)$ \\
\hline Tertile3 (>0.26) & 32 & 69 & 1.37 & $(0.72-2.57)$ & 2.88 & $(1.04-7.93)$ \\
\hline$P$ for trend & & & 0.37 & & 0.06 & \\
\hline \multicolumn{7}{|l|}{ whole Grains ${ }^{\mathrm{b}}$} \\
\hline Tertile $1(<0.51)$ & 32 & 67 & Ref & & Ref & \\
\hline Tertile2 (0.51-1.04) & 20 & 69 & 0.6 & $(0.31-1.16)$ & 0.7 & $(0.31-1.56)$ \\
\hline Tertile3 (>1.04) & 45 & 69 & 1.36 & $(0.77-2.40)$ & 0.43 & $(0.17-1.03)$ \\
\hline $\mathrm{P}$ for trend & & & 0.23 & & 0.35 & \\
\hline \multicolumn{7}{|l|}{ Dairy $^{\mathrm{a}}$} \\
\hline Tertile $1(<0.61)$ & 31 & 65 & Ref & & Ref & \\
\hline Tertile2 (0.61-0.95) & 32 & 69 & 0.97 & $(0.53-1.77)$ & 0.93 & $(0.38-2.23)$ \\
\hline Tertile3 $(>0.95)$ & 34 & 71 & 1 & $(0.55-1.81)$ & 0.99 & $(0.41-2.35)$ \\
\hline $\mathrm{P}$ for trend & & & 0.98 & & 0.99 & \\
\hline \multicolumn{7}{|l|}{ Total Protein Foods ${ }^{\mathrm{b}}$} \\
\hline Tertile $1(<1.40)$ & 33 & 67 & Ref & & Ref & \\
\hline Tertile2 (1.40-1.90) & 33 & 69 & 0.97 & $(0.53-1.74)$ & 0.47 & $(0.19-1.14)$ \\
\hline Tertile3 (>1.90) & 31 & 69 & 0.91 & $(0.50-1.63)$ & 0.5 & $(0.21-1.19)$ \\
\hline P for trend & & & 0.76 & & 0.11 & \\
\hline \multicolumn{7}{|l|}{ Seafood and Plant Proteins ${ }^{\mathrm{b}}$} \\
\hline Tertile $1(<0.18)$ & 18 & 68 & Ref & & Ref & \\
\hline Tertile2 (0.18-0.49) & 38 & 69 & 2.08 & $(1.08-3.99)$ & 0.37 & $(0.14-1.00)$ \\
\hline Tertile3 (>0.49) & 41 & 68 & 1.19 & $(1.19-4.35)$ & 1 & $(0.46-2.22)$ \\
\hline$P$ for trend & & & 0.01 & & 0.06 & \\
\hline
\end{tabular}


Table 4. Continued

\begin{tabular}{|c|c|c|c|c|c|c|}
\hline Variable in tertile & Case (n) & Control (n) & $\mathrm{OR}^{1}$ & $\mathrm{CI}$ & $\mathrm{OR}^{2}$ & $\mathrm{CI}$ \\
\hline \multicolumn{7}{|l|}{ Fatty Acids ${ }^{\mathrm{d}}$} \\
\hline Tertile $1(<1.67)$ & 81 & 67 & Ref & & Ref & \\
\hline Tertile2 (1.67-2.22) & 11 & 69 & 0.13 & $(0.06-0.26)$ & 0.09 & $(0.02-0.34)$ \\
\hline Tertile3 (>2.22) & 5 & 69 & 0.06 & $(0.02-0.15)$ & 0.12 & $(0.03-0.37)$ \\
\hline$P$ for trend & & & $<0.001$ & & $<0.001$ & \\
\hline \multicolumn{7}{|l|}{ Refined Grains ${ }^{\mathrm{c}}$} \\
\hline Tertile $1(<3.13)$ & 35 & 68 & Ref & & Ref & \\
\hline Tertile2 (3.13-4.81) & 34 & 69 & 0.95 & $(0.53-1.70)$ & 0.95 & $(0.39-2.30)$ \\
\hline Tertile3 $(>4.81)$ & 28 & 68 & 0.8 & $(0.43-1.45)$ & 1.17 & $(0.51-2.72)$ \\
\hline $\mathrm{P}$ for trend ined Grains & & & 0.47 & & 0.69 & \\
\hline \multicolumn{7}{|l|}{ Sodium $^{\mathrm{c}}$} \\
\hline Tertile $1(<1.84)$ & 3 & 68 & Ref & & Ref & \\
\hline Tertile2 (1.84-2.74) & 2 & 66 & 0.68 & $(0.11-4.24)$ & 0.66 & $(0.05-7.17)$ \\
\hline Tertile3 $(>2.74)$ & 92 & 71 & 29.73 & $(8.87-97.22)$ & 19.83 & $(4.40-88.10)$ \\
\hline$P$ for trend & & & $<0.001$ & & $<0.001$ & \\
\hline \multicolumn{7}{|l|}{ Empty calories ${ }^{\mathrm{e}}$} \\
\hline Tertile $1(<13.32)$ & 21 & 68 & Ref & & Ref & \\
\hline Tertile2 (13.32-18.76) & 46 & 69 & 2.15 & $(1.16-3.99)$ & 0.62 & $(0.23-1.62)$ \\
\hline Tertile3 $(>18.76)$ & 30 & 68 & 1.42 & $(0.74-2.74)$ & 1.49 & $(0.66-3.34)$ \\
\hline$P$ for trend & & & 0.75 & & 0.94 & \\
\hline
\end{tabular}

1, crude odds ratio; 2 , odds ratio adjusted for energy intake (continuous), hypertension (yes or no), diabetes (yes or no), smoking (yes or no), BMI (continuous), and waist circumstance (continuous); a, Density measure calculated as daily cups per 1,000 kcal; b, Density measure calculated as daily ounces per $1,000 \mathrm{kcal}$; c, Density measure calculated as daily grams per 1,000 kcal; d, Ratio of poly- and monounsaturated fatty acids to saturated fatty acids; e, total calories from solid fat, alcoholic beverages, and added sugar expressed as percent of total kcal.

be consistent with those reported by other studies which found statistically significant inverse association between PC risk and intake of legumes ref, beans ref, peas/beans/ lentils, and nut (Mills et al., 1989; Jain et al., 1999; Kolonel et al., 2000). The constituents of plant proteins that have anticarcinogenic properties and can be considered potentially protective against cancer could potentially account for a protective effect include fiber, protease inhibitors (Rao and Sung, 1995; Kennedy, 1998), saponins (Rao and Sung, 1995), inositol hexaphosphate (Fournier

Table 5. A Comparison between the Case and Control Groups Based on Daily Intake and Score Distribution of the Mediterranean-Style Dietary Pattern Score (MSDPS) Components; a Case-Control Study of PC in Iran.

\begin{tabular}{|c|c|c|c|c|c|c|c|}
\hline \multirow{2}{*}{$\frac{\text { MSDPS }}{\text { compone }}$} & \multicolumn{4}{|c|}{ Intake distribution } & \multicolumn{3}{|c|}{ MSDPS scores distribution } \\
\hline & Unit & Case (97) & Control (205) & pvalue & Case (95) & Control (205) & Pvalue* \\
\hline MSDPS total & - & - & - & & $26.20(18.98-35.94)$ & $24.49(19.20-33.63)$ & 0.44 \\
\hline Whole grain & Cup/daily & $2.39(0.70-5.00)$ & $1.83(1.09-2.89)$ & 0.15 & $2.99(0.88-6.25)$ & $2.28(1.37-3.61)$ & 0.12 \\
\hline Fruits & Cup/daily & $3.40(1.83-4.97)$ & $4.31(2.86-6.14)$ & $<0.001$ & $7.92(6.11-8.58)$ & $8.29(7.39-8.72)$ & 0.003 \\
\hline Vegetables & Cup/daily & $2.22(1.64-3.44)$ & $3.27(2.43-4.61)$ & $<0.001$ & $3.72(2.74-5.74)$ & $5.40(4.02-7.47)$ & $<0.001$ \\
\hline Dairy & Cup/daily & $2.14(1.52-2.84)$ & $2.27(1.49-3.20)$ & 0.29 & $8.42(7.01-8.80)$ & $8.34(7.30-8.69)$ & 0.34 \\
\hline $\begin{array}{l}\text { Fishe and } \\
\text { other seafood }\end{array}$ & $60 \mathrm{gr} /$ weekly & $1.48(0.68-2.79)$ & $0.94(0.36-2.60)$ & 0.21 & $2.48(1.14-4.67)$ & $1.55(0.60-4.41)$ & 0.17 \\
\hline Poultry & $60 \mathrm{gr} /$ weekly & $2.83(1.41-4.25)$ & $1.06(0.21-2.60)$ & $<0.001$ & $7.08(3.54-8.85)$ & $2.66(0.54-6.50)$ & $<0.001$ \\
\hline $\begin{array}{l}\text { Olives, } \\
\text { legumes, nuts }\end{array}$ & Cup/weekly & $4.79(2.60-9.78)$ & $5.20(2.67-8.32)$ & 0.9 & $7.36(5.58-8.40)$ & $7.70(5.94-8.56)$ & 0.43 \\
\hline Potatoes & Cup/weekly & $0.78(0.43-1.59)$ & $1.60(0.78-2.80)$ & $<0.001$ & $2.63(1.43-5.32)$ & $5.25(2.62-7.99)$ & $<0.001$ \\
\hline eggs & weekly & $2.08(1.04-3.16)$ & $0.99(0.01-2.99)$ & $<0.001$ & $6.92(3.46-7.89)$ & $3.32(0.04-7.99)$ & $<0.001$ \\
\hline sweets & Oz/ weekly & $0.94(0.41-2.02)$ & $0.95(0.36-2.69)$ & 0.48 & $10.00(10.00-10.00)$ & $10.00(10.00-10.00)$ & 0.051 \\
\hline Red meat & $60 \mathrm{gr} /$ mounthly & $16.85(7.20-27.07)$ & $23.35(12.22-3417)$ & $<0.001$ & $5.78(3.23-8.19)$ & $4.16(1.45-6.94)$ & $<0.001$ \\
\hline Olive oil & daily & $0.17(0.00-0.60)$ & $0.00(0.00-0.16)$ & $<0.001$ & - & - & $<0.001$ \\
\hline
\end{tabular}

* P-value estimated using Mann-Whitney; 1, Data are presented as median (5th, 95th percentile); 2, Total MSDPS was the sum of 12 components standardized to a $0-100$ scale and weighted to the proportion of daily energy intake from Mediterranean diet foods. 
et al., 1998), phytosterols (Steinmetz and Potter, 1996) and g-tocopherol (Steinmetz and Potter, 1996). We also found an association between other components and PC risk. The intake of fruits and vegetables, the components of both MED and HEI-2010, was inversely associated with PC risk. Previous reports also indicated that vegetables intake was associated with reduced PC risk (Kolonel et al., 2000; Takachi et al., 2009; Umesawa et al., 2014). There are several possible mechanisms for the inverse association between vegetable intake and risk of PC. For instance, vegetable components, including isothiocyanates and glucosinolates activate phase 2 enzyme, detoxificating carcinogen and stimulating cancer cell apoptosis (Hayes et al., 2008; Ho et al., 2009).

The results on the association between fruit intake and PC risk are contrasting. In some studies, fruits intake was associated with a reduced risk of cancer (Mills et al., 1989). While other studies showed that fruit consumption did not have any effects on cancer risk (Severson et al., 1989; Schuurman et al., 1998). Evidence for the protective effect of fruits is limited and the reported findings are contradictory (Umesawa et al., 2014). Previous reports highlighted increased risk of PC following animal fat and saturated fat consumptions (Le Marchand et al., 1994; Pelser et al., 2013). In the current study, an inverse association between higher ratio of USFA/ SFA intake and PC risk was found. The prospective studies showed that SFA intake was related to the risk of advanced or fatal PC (Pelser et al., 2013). This increased effect can be due to the effect of SFA on the level of sex hormones. Hill et al., (1979) showed that reducing the consumption of animal fats and replacing them with vegetable oils led to lower levels of estrogens and androgens.

Case-control studies are subject to limitations that should be considered in interpreting their results. First, the possibility of selection bias cannot be avoided in retrospective case-control studies. In addition, our controls might also have nutritional problems, which could dilute the association of dietary intakes and risk of PC due to sharing of the exposure. However, we preferred hospital controls (opposed to community controls) due to their higher participation and cooperation rates and to avoid selection bias. The present study had several strengths. First, the participation rate in this study was high. Second, patients whose disease was diagnosed during previous 6 months were registered in order to reduce the possibility of recall bias. This is due to the fact that dietary data collected at the time of disease ascertainment might not truly reflect past intakes or intakes during the development of disease. Third, we used a valid FFQ to reduce measurement error.

In conclusion, the present study was designed to determine the effect of diet quality on PC risk. The result of this investigation showed that healthy diet based on dietary guidelines for American 2010 can reduce the risk of PC. These findings enhance our understanding of the effects of dietary quality on PC risk. Further investigation on dietary indices is strongly recommended. Considerably more work is needed to be done to determine the best dietary recommendation for cancer prevention.
The authors declare that there is no conflict of interests regarding the publication of this paper.

\section{Acknowledgments}

We are grateful to all field investigators, staff, and participants. This study was supported by a grant from national nutrition and food technology research institute, Shahid Beheshti University of Medical Sciences, Tehran, Iran.

Note!!! It is very important to cite the references according to the journal rules and format.

Names of journals in your reference must be corrected. Please use "standard abbreviation names the journals".

\section{References}

Ambrosini GL, Fritschi L, De Klerk NH, et al (2008). Dietary patterns identified using factor analysis and prostate cancer risk: a case control study in Western Australia. Annals of epidemiology, 18, 364-70. (False)

Ambrosini GL, Fritschi L, De Klerk NH, et al (2008). Dietary patterns identified using factor analysis and prostate cancer risk: a case control study in Western Australia. Ann Epidemiol, 18, 364-70. (True)

Augustsson K, Michaud DS, Rimm EB, et al (2003). A prospective study of intake of fish and marine fatty acids and prostate cancer. Eaneer Epidenniology and Prevention Biomarkers, 12, 64-7.

Ax E, Garmo H, Grundmark B, et al (2014). Dietary patterns and prostate cancer risk: report from the population based ULSAM cohort study of Swedish men. Autrition and eaneer, 66, 77-87.

Baade PD, Youlden DR, Krnjacki LJ (2009). International epidemiology of prostate cancer: geographical distribution and secular trends. Moleetlar nutrition \& food researeh, $53,171-84$.

Bosire C, Stampfer MJ, Subar AF, et al (2013). Index-based dietary patterns and the risk of prostate cancer in the NIH-AARP diet and health study. Ameriean journal of epidemiology, 177, 504-13.

Bowman S, Clemens J, Friday J, et al (2014). Food Patterns Equivalents Database 2011-12: Methodology and User Guide. Worldwide Web Site: Food Surveys Research Group.

Chavarro JE, Stampfer MJ, Hall MN, et al (2008). A 22-y prospective study of fish intake in relation to prostate cancer incidence and mortality. The Ameriean journal of elinieal nutrition, 88, 1297-303.

Chavarro JE, Stampfer MJ, Li H, et al (2007). A prospective study of polyunsaturated fatty acid levels in blood and prostate cancer risk. Caneer Epidemiology and Prevention Biomarkers, 16, 1364-70.

Esfahani FH, Asghari G, Mirmiran P, et al (2010). Reproducibility and relative validity of food group intake in a food frequency questionnaire developed for the Tehran Lipid and Glucose Study. Journal of epidemiology, 20, 150-8.

Faust T, Lee E, Redfern J, et al (1989). Effect of prostaglandin $\mathrm{F} 3 \alpha$ on gastric mucosal injury by ethanol in rats: Comparison with prostaglandin F2 $\alpha$. Prostaglandins, 37, 493-504.

Fournier DB, Erdman J, Gordon GB (1998). Soy, its components, and cancer prevention: a review of the in vitro, animal, and human data. Eaneer Epidemiology and Prevention Biomarkers, 7, 1055-65. 
Ghaffarpour M, Houshiar-Rad A, Kianfar H (1999). The manual for household measures, cooking yields factors and edible portion of foods. Tehran: Nashre Olume Keshavarzy, 7, 213.

Ghosh J, Myers CE (1997). Arachidonic acid stimulates prostate cancer cell growth: critical role of 5-lipoxygenase. Biochemieal and biophysieal researeh eommunieations, 235, 418-23.

Giovannucci E, Rimm EB, Liu Y, et al (2002). A prospective study of tomato products, lycopene, and prostate cancer risk. J Natl Cancer Inst, 94, 391-8.

Grubb RL, 3rd, Kibel AS (2007). Prostate cancer: screening, diagnosis and management in 2007. Mo Med, 104, 408-13; quiz 13-4.

Guenther PM, Casavale KO, Reedy J, et al (2013). Update of the healthy eating index: HEI-2010. Journal of the Aeademy of Nutrition and Dieteties, 113, 569-80.

Guenther PM, Kirkpatrick SI, Reedy J, et al (2014). The Healthy Eating Index-2010 is a valid and reliable measure of diet quality according to the 2010 Dietary Guidelines for Americans. The Journal of nutrition, jn. 113.183079.

Hayes JD, Kelleher MO, Eggleston IM (2008). The cancer chemopreventive actions of phytochemicals derived from glucosinolates. European journal of nutrition, 47, 73-88.

Hill P, Wynder EL, Garbaczewski L, et al (1979). Diet and urinary steroids in black and white North American men and black South African men. Cancer Res, 39, 5101-5.

Ho E, Clarke JD, Dashwood RH (2009). Dietary sulforaphane, a histone deacetylase inhibitor for cancer prevention. The Journal of nutrition, 139, 2393-6.

Hosseini-Esfahani F, Jessri M, Mirmiran P, et al (2010). Adherence to dietary recommendations and risk of metabolic syndrome: Tehran Lipid and Glucose Study. Metabolism, 59, 1833-42.

Hosseini M, SeyedAlinaghi S, Mahmoudi M, et al (2010). A case-control study of risk factors for prostate cancer in Iran. Acta Med Iran, 48, 61-6.

Jain MG, Hislop GT, Howe GR, et al (1999). Plant foods, antioxidants, and prostate cancer risk: findings from casecontrol studies in Canada. Nutrition and eaneer, 34, 173-84.

Kennedy AR (1998). The Bowman-Birk inhibitor from soybeans as an anticarcinogenic agent. The Ameriean journal of elinieal nutrition, 68, 1406S-12S.

Kolonel LN, Hankin JH, Whittemore AS, et al (2000). Vegetables, fruits, legumes and prostate cancer: a multiethnic case-control study. Caneer Epidemiology and Prevention Biomarkers, 9, 795-804.

Le Marchand L, Kolonel LN, Wilkens LR, et al (1994). Animal fat consumption and prostate cancer: a prospective study in Hawaii. Epidemiology, vol???, 276-82.

McGuire S (2011). US Department of Agriculture and US Department of Health and Human Services, Dietary Guidelines for Americans, 2010. Washington, DC: US Government Printing Office, January 2011. Advances in Nutrition: An International Review Journal, 2, 293-4.

Michels KB, Schulze MB (2005). Can dietary patterns help us detect diet-disease associations? Autrition researehreviews, $18,241-8$.

Mills PK, Beeson WL, Phillips RL, et al (1989). Cohort study of diet, lifestyle, and prostate cancer in Adventist men. Cancer, 64, 598-604.

Mitrou PN, Kipnis V, Thiébaut AC, et al (2007). Mediterranean dietary pattern and prediction of all-cause mortality in a US population: results from the NIH-AARP Diet and Health Study. Arehives of Internal Medieine, 167, 2461-8.

Moeller SM, Reedy J, Millen AE, et al (2007). Dietary patterns: challenges and opportunities in dietary patterns research. Journal of the Ameriean Dietetic Assoeiation, 107, 1233-9.
Möller E, Galeone C, Andersson TM-L, et al (2013). Mediterranean Diet Score and prostate cancer risk in a Swedish population-based case-control study. Journal of nutritional seienee, 2, pages???

Movahedi A, Roosta R (2000). Food composition table. Tehran: Shaheed Beheshti University of Medical Sciences.

Muller DC, Severi G, Baglietto L, et al (2009). Dietary patterns and prostate cancer risk. Eaneer Epidemiology and Prevention Biomarkers, 18, 3126-9.

Onvani S, Haghighatdoost F, Surkan P, et al (2017). Adherence to the Healthy Eating Index and Alternative Healthy Eating Index dietary patterns and mortality from all causes, cardiovascular disease and cancer: a meta-analysis of observational studies. Fournal of Human Nutrition and Dieteties, 30, 216-26.

Park Y, Mitrou PN, Kipnis V, et al (2007). Calcium, dairy foods, and risk of incident and fatal prostate cancer: the NIH-AARP Diet and Health Study. Ameriean journal of epidemiology, 166, 1270-9.

Pelser C, Mondul AM, Hollenbeck AR, et al (2013). Dietary fat, fatty acids, and risk of prostate cancer in the NIH-AARP diet and health study. Caneer Epidemiology and Prevention Biomarkers, 22, 697-707.

Pham T-M, Fujino Y, Kubo T, et al (2009). Fish intake and the risk of fatal prostate cancer: findings from a cohort study in Japan. Publie health nutrition, 12, 609-13.

Rao A, Sung M (1995). Saponins as anticarcinogens. The Journat of nutrition, 125, 717S.

Reedy J, Mitrou P, Krebs-Smith S, et al (2008). Index-based dietary patterns and risk of colorectal cancer: the NIH-AARP Diet and Health Study. Ameriean journal of epidemiology, $168,38-48$.

Rose DP (1997). Effects of dietary fatty acids on breast and prostate cancers: evidence from in vitro experiments and animal studies. The Ameriean journal of elinieal nutrition, $66,1513 \mathrm{~S}-22 \mathrm{~S}$.

Rumawas ME, Dwyer JT, Mckeown NM, et al (2009a). The development of the Mediterranean-style dietary pattern score and its application to the American diet in the Framingham Offspring Cohort. The Journal of nutrition, 139, 1150-6.

Rumawas ME, Meigs JB, Dwyer JT, et al (2009b). Mediterraneanstyle dietary pattern, reduced risk of metabolic syndrome traits, and incidence in the Framingham Offspring Cohort. The Ameriean journal of elinieal nutrition, 90, 1608-14.

Schuurman AG, Goldbohm RA, Dorant E, et al (1998). Vegetable and fruit consumption and prostate cancer risk: a cohort study in The Netherlands. Caneer Epidemiology and Prevention Biomatkers, 7, 673-80.

Severson RK, Nomura AM, Grove JS, et al (1989). A prospective study of demographics, diet, and prostate cancer among men of Japanese ancestry in Hawaii. Caneer researeh, 49, 1857-60.

Sofi F, Abbate R, Gensini GF, et al (2010). Accruing evidence on benefits of adherence to the Mediterranean diet on health: an updated systematic review and meta-analysis. The Ameriean journal of elinieal nutrition, 92, 1189-96.

Steinmetz KA, Potter JD (1996). Vegetables, fruit, and cancer prevention: a review. Fournal of the Ameriean Dietetie Association, 96, 1027-39.

Takachi R, Inoue M, Sawada N, et al (2009). Fruits and vegetables in relation to prostate cancer in Japanese men: the Japan Public Health Center-Based Prospective Study. Nutrition and eancer, 62, 30-9.

Trichopoulou A, Lagiou P, Kuper H, et al (2000). Cancer and Mediterranean dietary traditions. Caneer Epidemiology and Prevention Biomarkers, 9, 869-73.

Tseng M, Breslow RA, DeVellis RF, et al (2004). Dietary patterns 
and prostate cancer risk in the National Health and Nutrition Examination Survey Epidemiological Follow-up Study cohort. Caneer Epidemiology and Prevention Biomarkers, 13, 71-7.

Umesawa M, Iso H, Mikami K, et al (2014). Relationship between vegetable and carotene intake and risk of prostate cancer: the JACC study. British journal of eaneer, 110, 792-6.

Verberne L, Bach-Faig A, Buckland G, et al (2010). Association between the Mediterranean diet and cancer risk: a review of observational studies. Autrition and eaneer, 62, 860-70.

Wu K, Hu FB, Willett WC, et al (2006). Dietary patterns and risk of prostate cancer in US men. Eaneer Epidemiology and Prevention Biomarkers, 15, 167-71.

Zaccheo T, Giudici D, di Salle E (1998). Effect of early treatment of prostate cancer with the $5 \alpha$-reductase inhibitor turosteride in Dunning R3327 prostatic carcinoma in rats. Fhe Prostate, 35, 237-42.

Zhang L, Yang B-X, Zhang H-T, et al (2011). Prostate cancer: an emerging threat to the health of aging men in Asia. Asian journal of andrology, 13, 574.

\section{c) (i) (8)}

This work is licensed under a Creative Commons AttributionNon Commercial 4.0 International License. 